\title{
Analysis of Bioelectrical Activity of the Athlete's Back Muscles During Special Exercises and Competition Actions in Such Power Sport as Mas- Wrestling
}

\author{
Artemenko T.G. \\ Churapcha State Institute of Physical Education and Sport \\ Churapcha, Russia \\ 2336964@ua.fm
}

\author{
Gotovtsev I.I. \\ Churapcha State Institute of Physical Education and Sport \\ Churapcha, Russia \\ rector@chgifkis.ru
}

\author{
Artemenko E.V. \\ Churapcha State Institute of Physical Education and Sport \\ Churapcha, Russia \\ ewa_12@ukr.net
}

\begin{abstract}
In the pre-competition period of training at the stage of higher sportsmanship, research studies were carried out concerning the bioelectric activity of back muscles of a maswrestler in the process of performing special exercises and in control competitive fights. The analysis of the interferential myogram of muscle activity allowed to distinguish special exercises by value of bioelectric activity. Mostly involved muscles and their group, which also have the greatest engagement in competitive motor action, were observed. One of the criteria on the basis of which the comparison was made was the parameter of the myogram - the total bioelectric muscle activity. For the analysis of the athlete's motor actions, were used the exercises of general physical orientation (deadlift with a dynamometer, squat with a weight, half-squat with a weight), the exercises of special physical orientation (weight lift of $100 \mathrm{~kg}$; weight lift of $100 \mathrm{~kg}$, the neck of which is located at the floor level; block lift at maximum effort) and competitive motor actions (triple- and double-support lift, lift in defense, the Ushnitskiy move and defense-hold). The homogeneity of two independent samples of the average total bioelectric activity results of mostly involved muscles was checked when performing eleven exercises of general and special physical orientation and competitive action.

The results of the study revealed that among all exercises, the maximum indicators of the total bioelectric activity of the upper trapezius muscle (Upper Trapezius) are shown only in the exercises "squat with a weight" $\left(1503,2 \pm 83,6 \mathrm{mV}^{*}\right.$ sec $)$ and "halfsquat with a weight" $(1345,3 \pm 56,9 \mathrm{mV} * \mathrm{sec})$.
\end{abstract}

Keywords-mas-wrestling; electromyography; total average bioelectric muscle activity; special exercises; competitive movement

\section{INTRODUCTION}

Power combat sport mas-wrestling represents the maximum dynamic efforts with globally involved topography of an athlete muscles in the conditions of statnamic strain. The structure and composition of the athletes motor movement is formed in specific conditions of internal and external environment, limited by space-time, power and leading motor qualities in the chosen sport. Fundamental theoretical theses on multilevel construction of the control system of human motor actions, proposed by N.A.Bernshtein [3], methodological foundations of the motor actions study in sports biomechanics, considered by A.A. Shalmanov, and other scientific works, confirmed by application studies on sports, pay attention to the need of following a strict sequence of motor skill development at each stage of sports improvement.

One of the first biomechanical principles, significant for the analysis of an athlete's movement, was considered to be the indicator of changing the power impulse, its alternation and preservation, the combination of separate impulses, and their counteraction [18]. The registration of these power factors was based on mechanical construction of rational use of forces in movement while doing physical exercises. The next stage in the biomechanical analysis is the study of specific character of the power impulse demonstration in the context of implementation of different target movements in sports [20].

Currently, the idea of studying movements is based on such concepts as structural biomechanical analysis of actions using quantitative characteristics $[1,10]$, including such terms as "kinematic mechanism", "biomechanism" [9].

A motor skill is considered to be developed, when it meets the requirements of sport already by the beginning of the higher sportsmanship stage, when it is time to maximize the implementation of the accumulated sports potential. 
Selection of training exercises on the basis of power demonstrations by a separate muscle as well as by a group of muscles, carrying the main motor load, corresponding to the motor action of the main competitive technique, will optimize the process of a special skill formation [6]. Studies of the power contribution of an individual muscle into the structure of motor action can be based on the fact of a close relationship between its strength and electrical impulse [10, 17].

One of the indicators of power manifestation level of muscles involved in motor action is the total area of electromyograms of their muscle activity. Comparison of training exercises and the main competitive method under the conditions, where it is possible to observe close motor characteristics between them (spatially-temporal), will allow to make reasonable assumptions about the choice of this or that exercise on the basis of the total muscular bioelectric activity indicator [15].

\section{LITERATURE REVIEW}

The well-known method of electromyography permits to determine the indicator of the total (interferential) bioelectric activity of the muscles involved in the motor action. History of this technique use has a certain accumulated experience in scientific and application research regarding solution to actual problems of determining reliable data of muscle tensions in various sports, and especially in those ones which require speed-and-power demonstration of motor qualities. I.M. Kozlov performed extensive research [8], aimed at studying the activation of muscles in the implementation of complex coordination exercises. In sports studies with the use of electroneuromiography method, significant results were obtained by R.M. Gorodnichev [5] in terms of examining the problems of muscle fatigue, functional readiness, mechanisms of regulation when performing special exercises.

The main orientation of physical qualities in competitive activity of a mas-wrestler is connected with the maximum manifestations of force. The increase in strength indicators is possible either by improving the process of controlling muscle activity, i.e. the development of intramuscular coordination, or by increasing the number of myofibrils in muscle fibers [13]. These two variants are realized by selection of special exercises intended for improvement of sports technique and motor qualities demonstrated while performing the main competitive movement. Estimation of such exercises, based on biomechanical indicators, must satisfy the principle of dynamic compliance (Yu.V. Verkhoshansky) [4, 7], i.e. coincide with the competitive action according to the following criteria: amplitude and direction of movement, accentuated area of the working movement amplitude, magnitude of the action force, speed of maximum force development, mode of muscle work.

The method of electromyography (EMG) allows to obtain the following indicators: relative magnitude of the action force, speed of maximum effort development and the mode of muscles operation. The used method has its application in sports research practice, despite its labor intensity and high cost of diagnostic equipment. According to human, time resources and cost of consumables EMG can be considered to be one of the most expensive methods. The integrated area of the myogram indirectly reflects the magnitude of the developed effort in an individual muscle [12].

The analysis of single potentials of motor units (PMU) in the conditions of minimum tension of a muscle, allows to investigate only low-threshold, mainly slow motor units. Obtaining of EMG with the activity of all motor units of the muscle is possible at its maximum spontaneous tension. In case of registration of bioelectric potentials of the involved muscle by the surface electrode, at maximum spontaneous tension, it is possible to receive an interferential EMG in the end. Herewith, due to the juxtaposition (interference) of potentials of motor units against each other, the possibility of visual marking of separate peak potentials of each motor unit is not available.

\section{RESEARCH METHODOLOGY}

In a series of experiments we carried out a comparative analysis with regards to EMG parameters of the athlete's back muscles - namely, master of sports of Russia in mas-wrestling (weight category of $70 \mathrm{~kg}$ ), 1990 year of birth, who is the 2018 world champion on mas-wrestling. On the basis of known examples of modeling in power sports and analysis of performing competitive power actions $[11,14]$, the athlete was offered to carry out motor actions in special training exercises which were similar to competitive ones in terms of load amount. The study used a group of general physical orientation exercises (deadlifts with a dynamometer, squat with a weight, half-squat with a weight), a group of special physical orientation exercises (weight lift and weight lift from the floor, each weighing $100 \mathrm{~kg}$, block lift at maximum effort) and a group of exercises of competition orientation (inner grip triple-support lift, outer grip double-support lift, inner grip defense lift, the Ushnitskiy move and defense-hold). The number of repetitions in each exercise was from 1 to 5 times. The weight amount in training exercises was selected individually, and the load was $100 \%$ of the individual maximum.

The technique of myogram registration and analysis of bioelectric activity of skeletal muscles by transmitting nerve impulses to them is as follows. In the process of measuring EMG was used an 8-channel hardware and software complex "Miocom" (developed by a specialized enterprise OKB "Rhythm", Taganrog, Russia) to evaluate electrical activity of muscles in the form of registration of electromyogram signals (EMG, also miogram). This device consists of a hardware unit connected to a laptop by USB-cable, surface recording electrodes with amplifiers and a grounding electrode (frequency range of input signals (by level - $3 \mathrm{~dB}$ ) is 15-600 $\mathrm{Hz}$, input voltage - 0-2 $\mathrm{mV}$, number of registration channels 8 , length of the signal lead $-1,5 \mathrm{~m}$, noise voltage, reduced to the input of no more than $1 \mathrm{mV}$, time constant of the RMS value - $100 \mathrm{~ms}$ ). The equipment allows to use the surface electrodes (REFF3010 size $41 \times 21 \mathrm{~mm}$, FIAB, Italy) for active movements. The existing external synchronization is determined for composing a unified timescale during video and other biomechanical investigations.

Electrodes were set on the athlete's skin in places of motor points localization. The place of their position and 
orientation was chosen with consideration to the studies known in this field $[16,19,20]$.

As for distinguishing separate phases of athlete movements and determination of muscles activity in these phases it is necessary to do video shooting synchronously with the record of electromyograms (EMG), it was required to use a synchronization device. The analysis of more than 5 sites of the registered myograms, with duration equal to a full phase of a motive action in each exercise, was carried out.

\section{RESULTS}

The processes of bioelectric conjugation of skeletal muscles of the mas-wrestler based on the co-ordination of contractile and relaxing modes, accompanying special exercises and competitive motor actions, showed the following.

In Table 1, the results of myograms with indicators of total bioelectric activity of eight back muscles of the wrestler during the execution of eleven motor actions are reflected. The table contains six exercises of general and special physical orientation: deadlift; weight lift of $100 \mathrm{~kg}$; weight lift of 100 $\mathrm{kg}$ from the floor level; block lift weighing $100 \mathrm{~kg}$; block lift at maximum effort and others. The specified training physical actions are similar to competitive activity in separate basic techniques of mas-wrestlers in terms of the motion structure: triple- and double-support lift, lift in defense, the Ushnitskiy move and defense-hold. Bioelectrical indicators during muscular activity in competitive actions of mas-wrestlers are presented in Table 1 according to differences not only in types of support, but also in types of gripping the pulling stick external and internal.

Training exercises from the group of general physical orientation, associated with the performance of squats and half-squats with loads of different weight, are basic for the development of total strength in many sports that require maximum demonstration of speed-and-power motor qualities. Therefore, it can be noted that the number of muscles and their absolute values are observed in this group with the highest results in comparison with the group of exercises of special physical orientation and competitive activity.

For example, it can be seen that among all exercises, the maximum results of the total bioelectric activity of the upper trapezius muscle (Upper Trapezius) are demonstrated only in the exercises "squat with a weight" $\left(1503,2 \pm 83,6 \mathrm{mV}^{*} \mathrm{sec}\right)$ and "semi-squat with a weight" $\left(1345,3 \pm 56,9 \mathrm{mV}^{*} \mathrm{sec}\right)$.

TABLE I. RESULTS OF THE TOTAL BIOELECTRIC ACTIVITY OF CERTAIN MUSCLES DURING SPECIAL EXERCISES AND COMPETITIVE ACTIONS

\begin{tabular}{|c|c|c|c|c|c|c|c|c|}
\hline \multirow{2}{*}{$\begin{array}{l}\text { Mas-wrestler } \\
\text { exercise }\end{array}$} & \multicolumn{8}{|c|}{$\begin{array}{l}\text { Muscle of the back and its total bioelectrical activity } \\
\text { (area of bioelectric activity, Sm, mV*sec) }\end{array}$} \\
\hline & $\begin{array}{l}\text { Upper } \\
\text { Trapezius }\end{array}$ & T2 Paraspinals & $\begin{array}{l}\text { Infraspinatu } \\
\mathrm{s}\end{array}$ & $\begin{array}{l}\text { Lower } \\
\text { Trapezius }\end{array}$ & $\begin{array}{l}\text { Latissimus } \\
\text { Dorsi }\end{array}$ & $\begin{array}{c}\text { T8 } \\
\text { Paraspinals }\end{array}$ & $\begin{array}{c}\text { T10 } \\
\text { Paraspinals }\end{array}$ & $\begin{array}{c}\text { L5 } \\
\text { Paraspinals }\end{array}$ \\
\hline & \multicolumn{8}{|c|}{ exercise of general physical orientation } \\
\hline $\begin{array}{l}\text { Deadlift, } \\
\text { dynamometry }\end{array}$ & $23,1 \pm 1,8$ & $18,5 \pm 4,7$ & $663,2 \pm 49,5$ & $127,3 \pm 11,4$ & $532,6 \pm 43,7$ & $152,6 \pm 9,6$ & $143,1 \pm 17,2$ & $10,6 \pm 1,7$ \\
\hline $\begin{array}{l}\text { Squat with } \\
\text { a weight }\end{array}$ & $1503,2 \pm 83,6$ & $626,7 \pm 57,1$ & $153,6 \pm 12,7$ & $901,3 \pm 47,3$ & $25,6 \pm 3,7$ & $746,5 \pm 65,8$ & $225,4 \pm 31,6$ & $231,7 \pm 19,7$ \\
\hline \multirow[t]{2}{*}{$\begin{array}{l}\text { Half-squat with } \\
\text { a weight }\end{array}$} & $1345,3 \pm 56,9$ & $518,5 \pm 51,2$ & $217,2 \pm 34,8$ & $894,7 \pm 76,9$ & $27,8 \pm 3,7$ & $493,2 \pm 45,2$ & $198,4 \pm 26,8$ & $228,6 \pm 18,4$ \\
\hline & \multicolumn{8}{|c|}{ exercise of special physical orientation } \\
\hline Weight lift, $100 \mathrm{~kg}$ & $430 \pm 33,2$ & $293 \pm 23,4$ & $92 \pm 5,6$ & $763,5 \pm 86,3$ & $531,3 \pm 43,5$ & $474,2 \pm 34,8$ & $208,9 \pm 26,1$ & $269,3 \pm 32,5$ \\
\hline $\begin{array}{l}\text { Weight lift, from } \\
\text { the floor level, } 100 \\
\mathrm{~kg}\end{array}$ & $543,8 \pm 64,8$ & $331,2 \pm 40,1$ & $138,6 \pm 10,4$ & $155,3 \pm 13,6$ & $558,7 \pm 67,8$ & $658,7 \pm 74,9$ & $217 \pm 19,3$ & $188,5 \pm 14,2$ \\
\hline \multirow[t]{2}{*}{$\begin{array}{l}\text { Maximum effort } \\
\text { at block lift }\end{array}$} & $60,9 \pm 1,9$ & $15,4 \pm 2,8$ & $20,3 \pm 1,5$ & $598,1 \pm 76,9$ & $126,5 \pm 9,4$ & $235,9 \pm 13,0$ & $155,2 \pm 17,8$ & $104,6 \pm 6,8$ \\
\hline & \multicolumn{8}{|c|}{ competitive actions } \\
\hline $\begin{array}{l}\text { Inner grip triple- } \\
\text { support lift }\end{array}$ & $76,3 \pm 9,4$ & $79,3 \pm 5,9$ & $60,5 \pm 4,9$ & $445,4 \pm 38,2$ & $105,8 \pm 9,0$ & $143,6 \pm 11,8$ & $135,9 \pm 12,5$ & $344,5 \pm 53,2$ \\
\hline $\begin{array}{l}\text { Outer grip double- } \\
\text { support lift }\end{array}$ & $119,7 \pm 10,2$ & $102,2 \pm 8,1$ & $59,4 \pm 2,3$ & $564,7 \pm 49,3$ & $270,4 \pm 47,1$ & $391,3 \pm 41,5$ & $168,5 \pm 23,1$ & $133,8 \pm 21,1$ \\
\hline $\begin{array}{l}\text { Inner grip defense } \\
\text { lift }\end{array}$ & $114,5 \pm 34,1$ & $83,9 \pm 4,7$ & $35,8 \pm 2,9$ & $439,0 \pm 37,8$ & $134,9 \pm 9,3$ & $193,4 \pm 3,9$ & $141,8 \pm 8,3$ & $86,5 \pm 7,1$ \\
\hline Ushnitskiy move & $323,7 \pm 49,2$ & $145,3 \pm 12,8$ & $75,7 \pm 4,8$ & $78,5 \pm 5,9$ & $297,1 \pm 41,6$ & $617,9 \pm 32,6$ & $253,5 \pm 43,1$ & $109,7 \pm 17,8$ \\
\hline Defense-hold & $371,6 \pm 46,5$ & $86,9 \pm 4,3$ & $37,5 \pm 2,8$ & $618,2 \pm 74,6$ & $42,9 \pm 3,2$ & $238,7 \pm 34,6$ & $228,8 \pm, 47,3$ & $12,6 \pm 1,4$ \\
\hline
\end{tabular}

It should be mentioned, that in the group of special physical orientation exercises, the number of muscles with high indicators of total bioelectric activity in a separate motor action is fewer than in the group of general physical orientation and more than in the group of competitive actions. This agrees with the effect of muscles elastic forces on the efficiency of muscle work [2]. 
The results of total bioelectric activity of the muscles involved in the performance of competitive actions have certain features, which are different from the other two groups of exercises. Here we observe the maximum indicators of total bioelectric activity of specific muscles when performing a competitive action. In this case, the lower trapezius internal muscle (Lower Trapezius) in motor actions shows the highest results (from $439,0 \pm 37,8\left(\mathrm{mV}^{*} \mathrm{sec}\right)$ in competitive action "defense lift with internal grip", and up to $618,2 \pm 74,6$ $\left(\mathrm{mV}^{*} \mathrm{sec}\right)$ - "in defense-hold" exercise).

Table 2 shows the value of average total bioelectric activity of the eight most involved back muscles in eleven exercises of general, special physical development and competitive actions of the mas-wrestler.

To determine the reliability concerning the absence of differences between the results of exercises of general and special physical orientation (sample 1) and competitive actions of the mas-wrestler (sample 2), an analysis was conducted to verify homogeneity of the two independent samples using the Wilcoxon criterion. These calculations included operations described below. We combined the samples and arranged the data in ascending order. Put down all the results in ascending order with assignment a serial number to each result, thereby obtaining a rank sequence. Out of 11 received numbers of the rank sequence, 6 (namely $1,3,8,9,10,11$ ) belong to groups of the general and special physical orientation, and $5(2,4,5$, $6,7)$ - to competitive actions group. The observed value of Wilcoxon statistics is Kobserv. $=2+4+5+6+7=24$. The critical area is double-sided. Critical values of statistics for certain sizes of samples at $\alpha=0,05$ are 18 for the left border and 42 for the right one. The observed value does not fall into the critical area: Kright < Kobserv. $<$ Kleft. The main hypothesis is confirmed, therefore, at the significance level of 0,05 , it is possible to assert that the difference between the observed results of the total bioelectric activity of back muscles is insignificant, when performing exercises of general, special physical orientation and competitive actions of the maswrestler.

The data presented in Table 2 indicate the highest average total bioelectric activity of muscles during general physical exercises (squat with a weight - 4414,0 $\mathrm{mV}^{*} \mathrm{sec}$, halfsquat with a weight $-3923,7 \mathrm{mV} * \mathrm{sec}$ ) and exercises of special physical orientation (weight lift, $100 \mathrm{~kg}-3062,2 \mathrm{mV} * \mathrm{sec}$, weight lift from the floor level, $100 \mathrm{~kg}-2791,8 \mathrm{mV}^{*} \mathrm{sec}$ ) in comparison with competitive actions (the Unitskiy move $\left.1901,4 \pm 176,9 \mathrm{mV}^{*} \mathrm{sec}\right)$.

The value of average total bioelectric activity of mostly involved back muscles shows significant differences between groups of exercises and competitive actions of the maswrestler.

TABLE II. AVERAGE TOTAL BIOELECTRIC ACTIVITY OF MUSCLES DURING SPECIAL EXERCISES AND COMPETITIVE ACTIONS

\begin{tabular}{|c|c|c|}
\hline No. & $\begin{array}{c}\text { Group of exercises } \\
\text { and competitive actions }\end{array}$ & $\begin{array}{c}\text { Total electrical muscles } \\
\text { activity, } \\
\text { SEMG }\left(\mathrm{mV}^{*} \mathrm{sec}\right) \pm \mathrm{S}\end{array}$ \\
\hline & group of exercises of general physical orientation \\
\hline 1 & deadlift, dynamometry & $1671,0 \pm 81,3$ \\
\hline 2 & squat with a weight & $4414,0 \pm 147,5$ \\
\hline
\end{tabular}

\begin{tabular}{|c|c|c|}
\hline 3 & half-squat with a weight & $3923,7 \pm 129,5$ \\
\hline & exercise of special physical orientation \\
\hline 4 & weight lift, 100 kg & $3062,2 \pm 152,65$ \\
\hline 5 & $\begin{array}{c}\text { weight lift, from the floor level, } \\
100 \mathrm{~kg}\end{array}$ & $2791,8 \pm 89,2$ \\
\hline 6 & maximum effort at block lift & $1316,9 \pm 143,2$ \\
\hline \multicolumn{2}{|c|}{ competitive actions } \\
\hline 7 & inner grip triple-support lift & $1391,3 \pm 54,7$ \\
\hline 8 & outer grip double-support lift & $1810,1 \pm 169,3$ \\
\hline 9 & inner grip defense lift & $1229,8 \pm 131,5$ \\
\hline 10 & Ushnitskiy move & $1901,4 \pm 176,9$ \\
\hline 11 & defense-hold & $1625,0 \pm 87,4$ \\
\hline & Note: SEMG is an arithmetic mean value of the total bioelectric activity of mostly involved muscles; S \\
is a mean square deviation
\end{tabular}

is a mean square deviation

The exercises that are similar to competitive actions are discovered: inner grip triple-support lift $(1391,3 \pm 54,7$ $\left.\mathrm{mV}^{*} \mathrm{sec}\right)$ and inner grip defense lift $\left(1229,8 \pm 131,5 \mathrm{mV}^{*} \mathrm{sec}\right)$. Such training exercises also include deadlift $(1671,0 \pm 81,3$ $\left.\mathrm{mV}^{*} \mathrm{sec}\right)$ and maximum effort at block lift $(1316,9 \pm 143.2$ $\left.\mathrm{mV}^{*} \mathrm{sec}\right)$.

Weight lift $\left(3062,2 \pm 152,65 \mathrm{mV}^{*} \mathrm{sec}\right)$ and weight lift from the floor level $\left(2791,8 \pm 89,2 \mathrm{mV}^{*} \mathrm{sec}\right)$, each $100 \mathrm{~kg}$, correspond to the indicators of the total bioelectric activity of mostly involved muscles, among competitive actions these are outer grip double-support lift $\left(1810,1 \pm 169,3 \mathrm{mV}^{*} \mathrm{sec}\right)$ and Ushnitskiy move $(1901,4 \pm 176,9 \mathrm{mV} * \mathrm{sec})$.

\section{CONCLUSION}

The athlete performed motor actions of different directions of training influence: exercises of basic general physical development, exercises of special physical development and competitive actions. In the process of observing total bioelectric activity of both separate muscles and their mutual contribution to the implementation of the motor task, different results were revealed.

A characteristic feature of performing exercises of general and special physical orientation that distinguishes it from competitive actions is high values of bioelectric activity indicators of both certain muscles and their total mutual contribution. This is the evidence of a selective training effect of each exercise on a specific muscle group. The obtained results allow to select purposefully general and special physical exercises in accordance with the requirement of bioelectric muscular activity of competitive motor action.

Thus, we can point to the maximum results of the total bioelectric activity of the upper trapezius muscle (Upper Trapezius), which are demonstrated only in the exercises "squat with a weight" $\left(1503,2 \pm 83,6 \mathrm{mV}^{*} \mathrm{sec}\right)$ and "half-squat with a weight" $(1345,3 \pm 56,9 \mathrm{mV} * \mathrm{sec})$.

Among motor actions of competitive character, it is possible to observe maximum values of bioelectric activity of mostly involved muscles in fulfilling the Ushnitsky move $\left(1901,4 \pm 176,9 \quad \mathrm{mV}^{*} \mathrm{sec}\right)$ and double-support lift $\left(1810,1 \pm 169,3 \mathrm{mV}^{*} \mathrm{sec}\right)$. Minimum values of bioelectric activity of the same back muscles are registered when performing inner grip defense lift $\left(1229,8 \pm 131,5 \mathrm{mV}^{*} \mathrm{sec}\right)$. 
[12]Mikhailyuk M.P. Example of modeling the level of speed and strength training of weightlifters. Weightlifting: Yearbook. M. 1983, pp. 28-29.

\section{References}

[1] Anokhin P.K. Key issues of the theory of functional systems. Moscow: Nauka. 1980, 109.

[2] Aruin A.S., Volkov N.I., Zatsiorsky V.M. Influence of elastic forces of muscles on efficiency of muscular work. Human Physiology. 1977, vol. 3, pp. 1410-1415.

[3] Bernstein N.A. About dexterity and its development. Moscow: Physical Culture and Sport. 1991, 288.

[4] Bernstein N.A. Essays on physiology of movements and physiology of activity. Moscow: Medicine. 1966, 349.

[5] Verkhoshansky Yu.V. Fundamentals of special strength training in sport. Moscow: Soviet Sport. 2013, 216.

[6] Gorodnichev R.M. Sports electroneuromyography. Velikiye Luki: Publishing House of Velikiye Luki State Academy of Physical Culture. 2005, 227.

[7] Gorodnichev R.M. The Physiology of Power. Monograph. Moscow: Sport. 2016, 232.

[8] Zatsiorsky V.M. Biomechanics of the human motor apparatus. Moscow: Physical Culture and Sport. 1981, 143.

[9] Kozlov I.M. Biomechanical factors of the organization of sports movements. Monograph. State Academy of Physical Culture named after P.F. Lesgaft. Saint Petersburg. 1998, 141.

[10] Koryak Yu.A. Methods of investigation of the neuromuscular system in athletes. Institute of Medico-Biological Problems of the Russian Acad. of Sciences. M. 1992, 63.

[11] Kostyuchenko V.F. Methods of registration of muscles electrical activity when performing physical exercises (EMG). Scientific notes of the University named after P.F. Lesgaft. 2007, 9(31), pp. 52-56.
[13]Persons R.S. Theoretical basis for the interpretation of the electromyogram. Human Physiology. 1987, vol. 13, 4, pp. 659-673.

[14] Samsonov A.V. Phase portraits of the muscles. Theory and Practice of Physical Culture. 1993, 1, pp. 1-3.

[15] Tsipin L.L. Analysis of static positions when performing exercises in kettlebell lifting. Russian Journal of Biomechanics. 2017, vol. 21, 2, pp. 178-187.

[16] Tsipin L.L., Zakharov F.E. Comparative analysis of the exercises of speed and strength training of Greco-Roman wrestlers. Scientific notes of the University named after P. F.Lesgaft, 2012, 11(93), pp. 132-137.

[17]Florimond V. Basics of Surface Electromyography Applied to Psychophysiology. Thought Technology Ltd. Montreal, Canada. 2008.

[18] Fuglevand A.J., Winter D.A., Patla A.E. Models of recruitment and rate coding organization in motor unit pools. Journal of Neurophysiology. 1993, 70, pp. 2470-2488.

[19] Hochmuth G. Biomechanic sport movements. Berlin: Sportverlag, Berlin. $1981,208$.

[20]Krol H., Sobota G., Nawrat A. Location of electrodes and bioelectric activity of muscles. Book of Abstracts of $8^{\text {th }}$ International Sports Science Conference, Vilnius, 24-25 February 2006, Lithuania. 2006.

[21]Kim-Kimen A.A., Kuznetsova Z.M. The development of the ancestors traditions for the Yakut sports glory. The Russian Journal of Physical Education and Sport. 2018, 13(2), pp. 119-123. DOI: 10.14526/02_2018_319.

[22]Krol H., Sobota G., Nawrat A., Wilk M. Complex analysis of movement in evaluation of flat bench press performance. XXIV International Symposium of Sport Biomechanics, Salzburg. Austria. 2006, pp. 1-4. 